\title{
Optimal Waveform Design for Smart Noise Jamming
}

\author{
Xingyu $\mathrm{Xia}^{1, \mathrm{a}}$, Daoliang $\mathrm{Hao}^{2, \mathrm{~b}}$ and Xiaohe $\mathrm{Wu}^{3, \mathrm{c}}$ \\ ${ }_{1,2,3}$ Luoyang Electronic Equipment Test Centre, Luoyang, China \\ a13383886001@163.com, bdearlord@live.com, 51008713@qq.com
}

Keywords: Smart noise jamming; Homomorphic filtering; Kalman filtering; Styling; Mechanism

\begin{abstract}
Optimal jamming waveform design is an effective way to improve the efficiency of electronic countermeasure, which makes it the focus issue in the field of electronic warfare. With new jamming technology emerging, jamming waveforms present characteristic of smart and intelligent. Owing to it, the smart noise jamming is studied, modeling and simulation of smart noise jamming mechanism is researched. Based on homomorphic and Kalman filtering algorithm analysis, smart noise jamming waveforms design method is presented according to complex cepstrum of homomorphic filtering and divergence of Kalman filtering, which will provide reference for other related waveform design.
\end{abstract}

\section{Introduction}

Jamming and anti-jamming is a pair of contradiction, and optimal jamming type exists only under the specific restriction of anti-jamming. With the development of radar technology, jamming technology is constantly emerging and jamming type presents smart and efficient. Since the concept of smart noise jamming proposed, simulation and application of that achieves well development, smart noise jamming is the compound jamming technique to modern radar, which represents a compromise between deception and noise jamming. The advantage of smart noise jamming is that we can attain better power management. Basically, the jamming energy can be optimized by tailoring waveforms to match threat radar's center frequency and bandwidth.

In this paper, we focus on the question that how to design optimal waveforms of smart noise jamming against modern radar. Firstly, modeling and simulation of smart noise jamming is researched in terms of physical domains. Secondly, from the perspective of modern radar techniques, the homomorphic and Kalman filtering algorithm is analyzed in terms of data domains. Thirdly, the optimum waveforms design method is proposed according to complex cepstrum of homomorphic filtering and divergence of Kalman filtering.

\section{Mechanism of Smart Noise Jamming}

Algorithm of Smart Noise Jamming. Matched-filtering theory is important for modern radar,with which we can obtain the maximum signal-to-noise(SNR). Smart noise jamming algorithm achieves matched filter gain by modulation jamming pulse on radar signals. Smart noise jamming waveform is coherent with radar signal character, which can bring false targets deception jamming effect and noise cover jamming effect ${ }^{[1]}$. From output to input, jamming waveform should be time-dense, amplitude-random pulse train, as is shown in Eq.1.

$$
m(t)=\sum_{n=0}^{\infty} A_{n} \delta\left(t-\sum_{i=0}^{n} t_{i}\right)
$$

The jamming signal should be the pulse train convolving with radar signal. Smart noise jamming algorithm is given by 


$$
\left\{\begin{aligned}
y_{0}(t) & =h(t) \otimes s(t) \\
J(t) & =s(t) \otimes m(t)=s(t) \otimes \sum_{n=0}^{\infty} A_{n} \delta\left(t-\sum_{i=0}^{n} t_{i}\right) \\
y(t) & =h(t) \otimes J(t)=h(t) \otimes \sum_{n=0}^{\infty} A_{n} s\left(t-\sum_{i=0}^{n} t_{i}\right) \\
& =\sum_{n=0}^{\infty} h(t) \otimes A_{n} s\left(t-\sum_{i=0}^{n} t_{i}\right) \\
& =\sum_{n=0}^{\infty} A_{n} y_{0}\left(t-\sum_{i=0}^{n} t_{i}\right)
\end{aligned}\right.
$$

Where $s(t)$ is radar signal, $h(t)$ is matching filter, $J(t)$ is jamming signal, $y_{0}(t)$ is radar signal after matching filter, $y(t)$ is jamming signal after matching filter.

We can see from Eq.2 that $y(t)$ consists of multiple false targets, which fall behind the real target. Due to the coupling relation between time-delay and frequency-shift, as is shown in Eq.3, the position of false targets can be changed to cover the true target by appropriate frequency-shift ${ }^{[2]}$.

$$
S\left(f-f_{d}\right) \leftrightarrow s(t) e^{2 \pi j f_{d} t}
$$

Modeling and Simulation of Smart Noise Jamming. Take linear frequency modulation (LFM) signal of PD radar for example, the pulse width of LFM is $50 \mu \mathrm{s}$, bandwidth is $10 \mathrm{MHz}$, sampling frequency is $40 \mathrm{MHz}$. Convoluted interfere is Gaussian noise, which obeys $(0,1)$ distribution, and the noise width is $10 \mu \mathrm{s}$. Time-domain waveform of smart noise jamming is shown in Fig. 1, the magnitude-frequency of smart noise jamming waveform after matched filter is shown in Fig. 2.

The true target position is set to 1000 meters, the relative position of false targets and true target before frequency-shift is shown in Fig. 3, from which we can see that false targets only exist after the true target. Through shifting frequency by $1 \mathrm{MHz}$, false targets have covered the true target as is shown in Fig. 4.

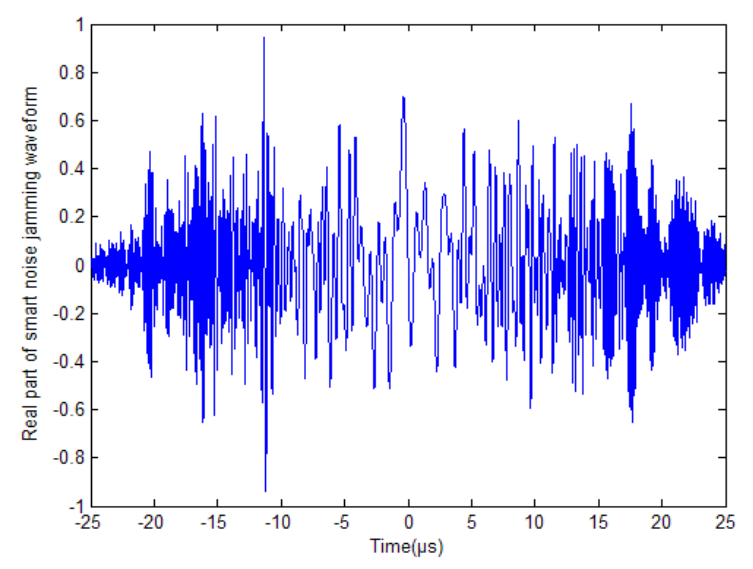

Figure 1. Time-Domain Waveform of Smart Noise Jamming 


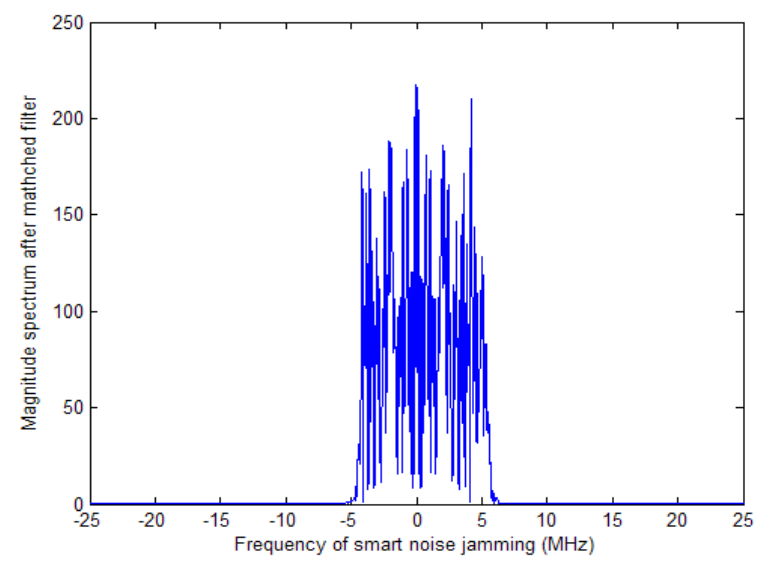

Figure 2. Magnitude-Frequency of Smart Noise Jamming

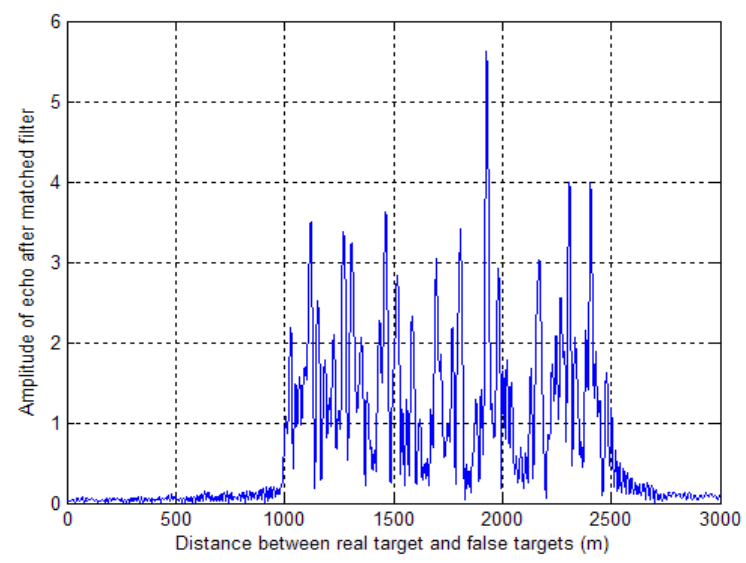

Figure 3. Relative Position of False Targets and True Target before Frequency-Shift

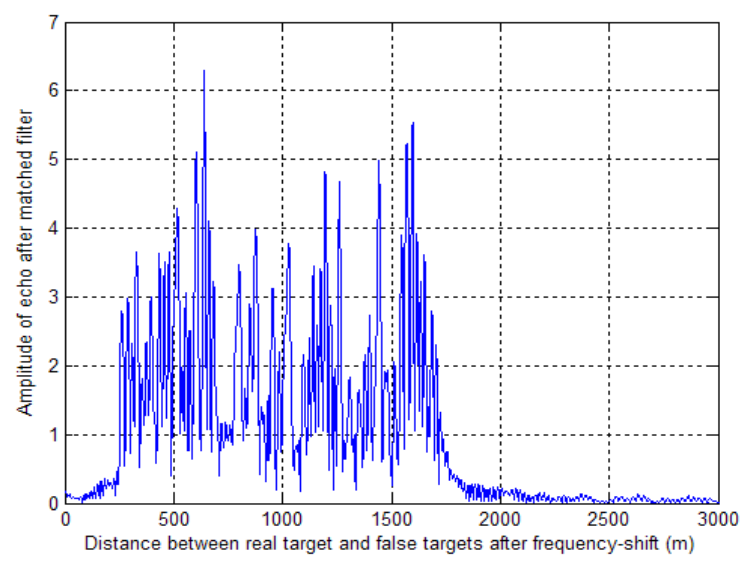

Figure 4. Relative Position of False Targets and True Target after Frequency-Shift

\section{Analysis of Radar Anti-Jamming Technology}

At present, the commonly used radar anti-jamming technology can be divided into the physical signal level and radar data level of anti-interference measures. Among the signal anti-jamming technology, there is frequency agility, pulse compression, sidelobe blanking sidelobe cancellation and so on ${ }^{[3][4]}$. They are not effective enough against smart noise jamming. For frequency agility radar, smart noise 
jamming is capable of distinguishing the agile frequency by wide-band receiver, and of quickly jamming by duplicating the radar signal exactly. Due to coherent with radar signal, interference signal can achieve the compression gain, making the radar difficult to distinguish true target and false target. According to sidelobe blanking technology theory, if it works on all the false targets, the main beam will miss target in virtue of excessive sidelobe blanking.

In the aspect of radar data anti-jamming technology, there is the homomorphic filtering, Kalman filtering for optimal estimation of target track, etc. Both of the filtering methods can form a certain inhibited ability to smart noise jamming. Based on the radar data anti-jamming technology, this paper carry out the problem of smart noise jamming optimal waveform design.

\section{Function Mechanism of Homomorphic Fitering and Kalman Filtering}

Function Mechanism of Homomorphic Filtering. Jamming modulation can be usually divided into superposition modulation, multiplicative modulation and convolution modulation, etc. Superposition modulation can be used to deal with linear filtering. In case of multiplicative modulation and convolution modulation, radar needs to convert the modulation into superposition form and remove the noise by linear filtering, which is the basic idea of homomorphic filtering ${ }^{[5]}$. Homomorphic filtering algorithm for deconvolution modulation can be described as Eq.4.

$$
\left\{\begin{array}{l}
x(n)=s(n) \otimes u(n) \\
X(z)=S(z) * U(z) \\
\ln X(z)=\ln S(z)+\ln U(z) \\
\hat{X}(z)=\ln X(z), \hat{S}(z)=\ln S(z), \hat{U}(z)=\ln U(z) \\
\hat{X}(z)=\hat{S}(z)+\hat{U}(z) \\
L^{-1}[\hat{X}(z)]=\hat{x}(n), L^{-1}[\hat{S}(z)]=\hat{s}(n), L^{-1}[\hat{U}(z)]=\hat{u}(n) \\
\hat{y}(n)=h(n) * \hat{x}(n)=\hat{s}(n) \\
\hat{Y}(z)=\hat{S}(z) \\
Y(z)=\exp (\hat{Y}(z))=\exp (\ln S(z))=S(z) \\
L^{-1}[Y(z)]=L^{-1}[S(z)]=s(n)
\end{array}\right.
$$

Where $\otimes$ represents convolution modulation, $s(n)$ is radar signal, $u(n)$ is jamming pulse.

Function Mechanism of Kalman Filtering. In order to realize tracking and measurement of moving target, the target data need to be extracted in a large amount of noise signal. Effective filtering algorithm is needed to be taken according to the characteristics of the target for improving the tracking accuracy. Kalman filtering belongs to linear minimum variance estimation for dynamic system's state sequence, which provides mathematical random estimation from measurement data that contains noise. The Kalman filtering algorithm is shown as Eq.5.

$$
\left\{\begin{array}{l}
\hat{s}_{k+1 / k+1}=\hat{s}_{k+1 / k}+K_{k+1}\left(z_{k+1}-H_{k+1} \hat{s}_{k+1 / k}\right) \\
\hat{s}_{k+1 / k}=\Phi_{k} \hat{s}_{k / k}+B_{k} u_{k} \\
\hat{P}_{k+1 / k}=\Phi_{k} \hat{P}_{k / k} \Phi_{k}^{T}+Q_{k} \\
K_{k+1}=\hat{P}_{k+1 / k} H_{k+1}^{T}\left(H_{k+1} \hat{P}_{k+1 / k} H_{k+1}^{T}+R_{k+1}\right)^{-1} \\
\hat{P}_{k+1 / k+1}=\left(I-K_{k+1} H_{k+1}\right) \hat{P}_{k+1 / k}
\end{array}\right.
$$




\section{Optimal Jamming Waveform Design Method}

Homomorphic filtering is the main algorithm to demodulate multiplicative modulation and convolution modulation, in which cepstrum analysis can be applied to separate smart noise jamming signal and target signal. As to optimal estimation of target tracking, Kalman filtering can also be applied to target separation. The two algorithms can be used to deal with the pulse train of smart noise jamming signal after matched filtering.

To design the optimal waveform of smart noise jamming equals to design $A_{n}$ and $t_{i}$ in Eq.1, which can make homomorphic filtering extract target parameter to the minimum degree and Kalman filtering can't predict the precise estimation. Therefore, after overcoming the limitations of physical domain, the optimal waveform design of smart noise jamming becomes a design issue in data domain.

Design Method Based on Cepstrum Analysis of Homomorphic Filtering. Homomorphic filtering can convert nonlinear problem into a linear one. After the processing of homomorphic filtering for radar signal, which containing smart noise jamming signal, the data's characteristics can be described by Eq.6.

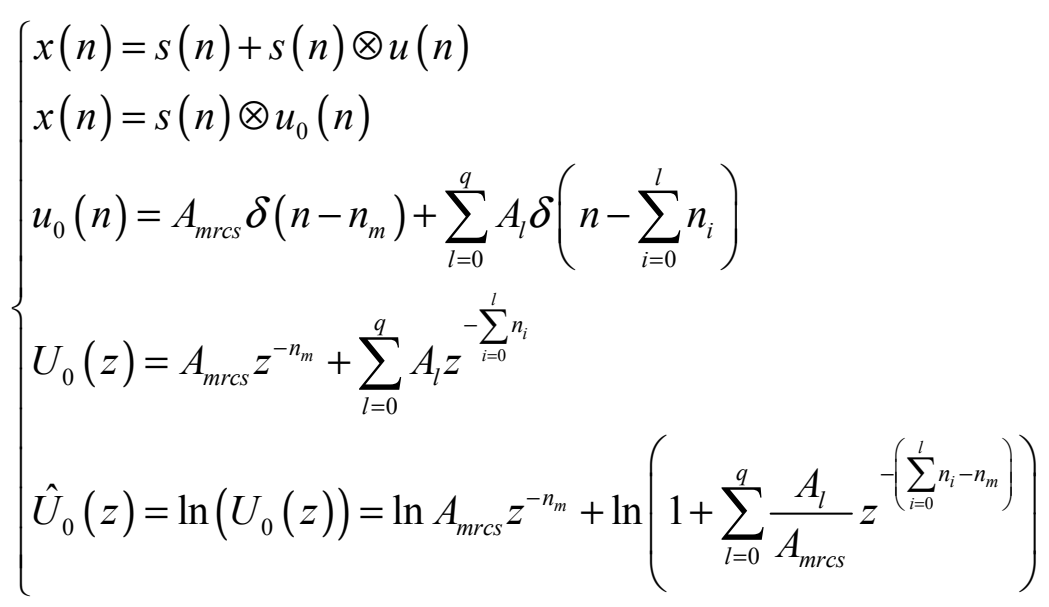

How to design linear system's weight function $h(n)$ for separating cepstrum $\hat{s}(n)$ and $\hat{u}_{0}(n)$ is the difficulty of homomorphic filtering algorithm. Typical linear constant frequency system spectral characteristics are short pass, long pass and comb. Due to the difference in time series of cepstrum, $h(n)$ can be designed as overlapping and non-overlapping function to suppress smart noise jamming.

After frequency shift, smart noise jamming could have overlapping effect in cepstrum. If the amplitude ratio of false target and true target is greater than $1, \hat{U}(z)$ will not converge. But if the ratio is smaller than $1, h(n)$ can't separate target signal either. Nevertheless, target signal can be extracted from cepstrum by the moment difference between the true target and the false one, which is shown in Fig. 5. The false targets need to form a shielding effect around true target, so the moment difference will be far smaller than target point's moment. Then, long pass $h(n)$ can be used to get rid of false target information.

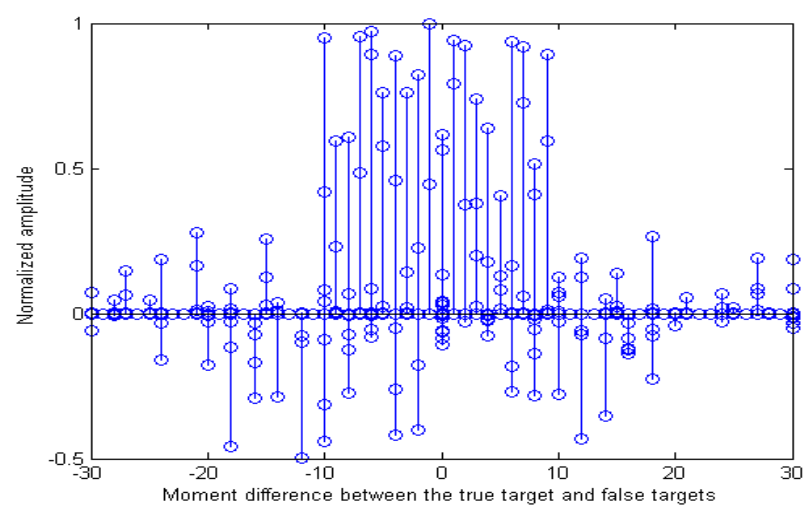

Figure 5. Cepstrum Map of Relative Relationship between True Target and False Targets 
To design waveform of smart noise jamming, combination method of sparse false targets and dense false targets could be applied, which will create dense false targets near the true target, while generate sparse false targets in where far from the true one. Distribution of the false target is designed as Fig. 6, which can decrease the performance of target detection.

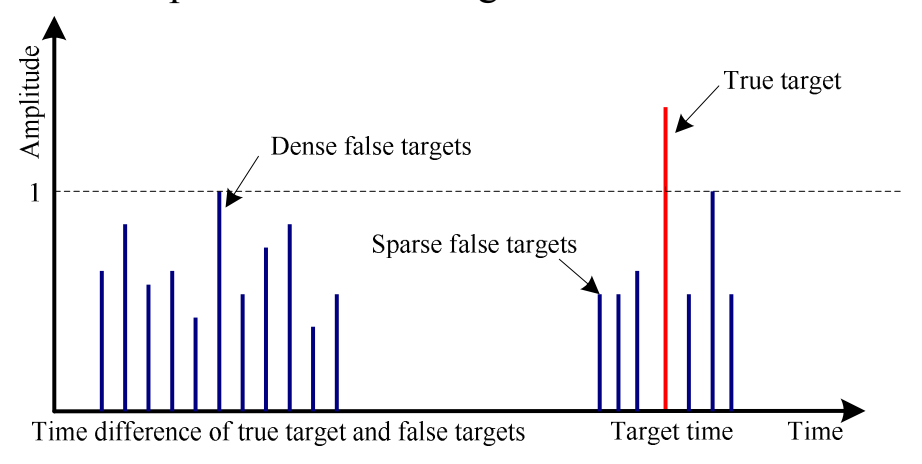

Figure 6. False Targets Distribution Design Sketch Map

Design Method Based on Divergence of Kalman Filtering. Data rationality test is an important part of radar data processing. Due to the impact of various accidental factors, serious deviation will be caused from the value of true target, which called outliers ${ }^{[6]}$. Kalman filtering can be used to deal with outliers. The false targets generated by smart noise jamming can be regarded as outliers. As long as the false target influenced the effectiveness of Kalman filtering, we can take this as the basis for the waveform design of smart noise jamming. Fig. 7 is the sketch map of containing multiple false targets detection, where $\Delta A$ and $\Delta d$ can be considered as the $A_{n}$ and $t_{i}$ in Eq. 1.

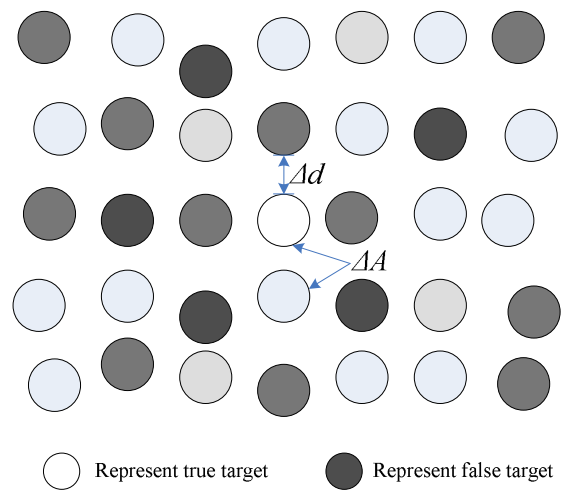

Figure 7. Data Detection of Multiple False Targets and True Target

According to Kalman filtering algorithm, the prediction residual $V_{k+1}$ can be deduced by $K$ moment state parameters. Based on statistical theory, if $V_{k+1}(i)$ meets Eq.7, the detection probability of outliers can reach $95 \%$. While if the detection probability of outliers is at $4.5 \%$, the outliers can be considered as true target. The probability distribution of $A_{n}$ and $t_{i}$ could be designed as the correlation function of Kalman residual, which is shown as Fig. 8.

$$
\left\{\begin{array}{l}
v_{k+1}=Z_{k+1}-H_{k+1} X_{k+1 / k} \\
E\left(v_{k+1} v_{k+1}^{\prime}\right)=H_{k+1} \hat{P}_{k+1 / k} H_{k+1}^{\prime}+R_{k+1} \\
v_{k+1}(i) \leq C \sqrt{\left[H_{k+1} \hat{P}_{k+1 / k} H_{k+1}^{\prime}+R_{k+1}\right]_{i, j}} \\
\left|v_{k+1}(i)\right| \leq C \sqrt{\left[H_{k+1} \hat{P}_{k+1 / k} H_{k+1}^{\prime}+R_{k+1}\right]_{i, j}}+2 \sqrt{\left[H_{k+1} \hat{P}_{k+1 / k} H_{k+1}^{\prime}\right]_{i, j}}
\end{array}\right.
$$




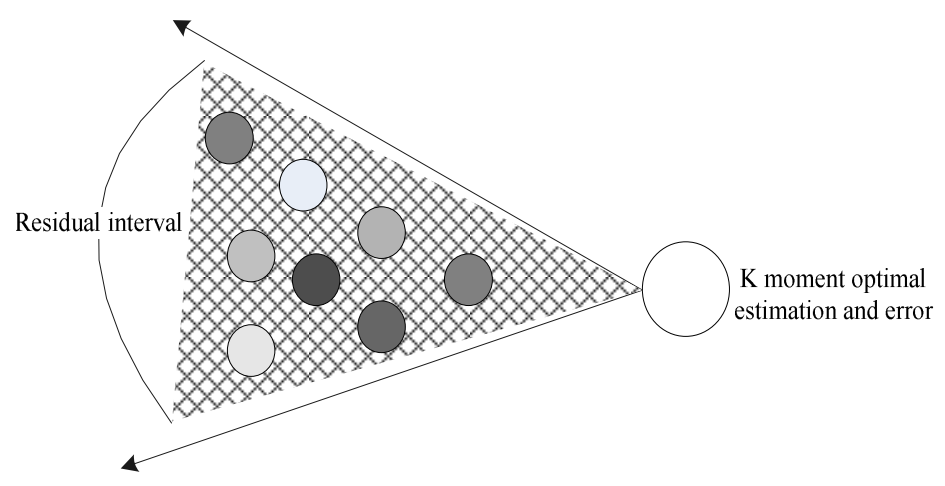

Figure 8. Design Interval of the False Targets for Smart Noise Jamming

\section{Summary}

Smart noise jamming is a hot spot in the research of radar jamming technology. The effect of smart noise jamming is studied from the physical signal level. From radar data processing level, the optimal waveform design method is proposed in the light of cepstrum of homomorphic filtering and divergence of Kalman filtering. The role and effectiveness of the data anti-jamming technology will become increasingly prominent, which will be the development direction of radar countermeasure field.

\section{References}

[1] J. Qiu and L.Y. QIU, Essential signification of smart noise jamming, Modern Defence Technology, vol. 40, pp. 132-136, Jun 2012.(In Chinese)

[2] L. Guo,H. Li and Q.S. Li, Interrupted sampling smart jamming method for coherent radar, Modern Defence Technology, vol. 41, pp. 111-116, Jun 2013.(In Chinese)

[3] Y.Y. Meng, H. Wu, S.Y. Cheng and J.N. Chi, Effecticeness evaluation of smart noise jamming against active radar seeker, Journal of Projectiles, Rockets, Missiles and Guidence vol. 35, pp. 173-176, Feb 2015.(In Chinese)

[4] X.J. Dai and C.H. Xu, Computer simulation of jamming PD radar, Modern Electronics Technique, vol.35, pp.39-41, Sep 2013.(In Chinese)

[5] G.S. Hu, Theory, Algorithm and Implementation for Digital Signal Processing. Beijing: Tsinghua University Press, 2007.(In Chinese)

[6] Y. He, J.J. Xiu, J.W. Zhang and X. Guan, Radar data processing with application. Beijing: Publishing House of Electronics Industry , 2009.(In Chinese) 